\title{
Human Wharton's jelly mesenchymal stem cells promote skin wound healing through paracrine signaling
}

\author{
Anna I Arno ${ }^{1,2}$, Saeid Amini-Nik², Patrick H Blit², Mohammed Al-Shehab², Cassandra Belo², Elaine Herer \\ Col Homer Tien ${ }^{4}$ and Marc G Jeschke ${ }^{2^{*}}$
}

\begin{abstract}
Introduction: The prevalence of nonhealing wounds is predicted to increase due to the growing aging population. Despite the use of novel skin substitutes and wound dressings, poorly vascularized wound niches impair wound repair. Mesenchymal stem cells (MSCs) have been reported to provide paracrine signals to promote wound healing, but the effect of human Wharton's jelly-derived MSCs (WJ-MSCs) has not yet been described in human normal skin. The aim of this study is to examine the effects of human WJ-MSC paracrine signaling on normal skin fibroblasts in vitro, and in an in vivo preclinical model.
\end{abstract}

Methods: Human WJ-MSCs and normal skin fibroblasts were isolated from donated umbilical cords and normal adult human skin. Fibroblasts were treated with WJ-MSC-conditioned medium (WJ-MSC-CM) or nonconditioned medium.

Results: Expression of genes involved in re-epithelialization (transforming growth factor- $\beta 2$ ), neovascularization (hypoxia-inducible factor-1a) and fibroproliferation (plasminogen activator inhibitor-1) was upregulated in WJ-MSCCM-treated fibroblasts $(P \leq 0.05)$. WJ-MSC-CM enhanced normal skin fibroblast proliferation $(P \leq 0.001)$ and migration $(P \leq 0.05)$, and promoted wound healing in an excisional full-thickness skin murine model.

Conclusions: Under our experimental conditions, WJ-MSCs enhanced skin wound healing in an in vivo mouse model.

\section{Introduction}

Nonhealing or chronic wounds represent an increasingly prevalent and costly public health issue. As a consequence of longer lifespans due to improvements in acute care, the number of patients who suffer diabetes and other chronic aging-related diseases is growing considerably. There is a link with aging-associated diseases and wound healing impairment [1]. On the other hand, other wound healing challenges also arise at any age because of accidents, burns and other traumatic injuries.

Mesenchymal stem cells (MSCs) appear to emerge as a promising wound healing therapy. Paracrine signaling, such as the release of factors that promote angiogenesis, immunomodulation and recruitment of endogenous

\footnotetext{
* Correspondence: marc.jeschke@sunnybrook.ca

${ }^{2}$ Ross Tilley Burn Centre and Sunnybrook Research Institute, Sunnybrook Health Sciences Centre, University of Toronto, 2075 Bayview Avenue, Toronto, ON M4N 3M5, Canada

Full list of author information is available at the end of the article
}

tissue stem/progenitor cells, as well as differentiation, have been described as possible mechanisms underlying the positive wound healing effects of MSCs [2,3]. From the different available sources of MSCs, the umbilical cord represents a cost-effective, productive, feasible, accepted, and universal source to isolate MSCs, and is considered advantageous compared with bone marrowderived mesenchymal stem cells (BM-MSCs) and adipose-derived MSCs for some researchers [4]. Previous studies with umbilical cord Wharton's jelly-derived mesenchymal stem cells (WJ-MSCs) have demonstrated that they represent a high-yield source of young, nontumorigenic and immunomodulatory cells that may be allotransplanted to regenerate liver, heart, bone, cartilage, fat, pancreas, neural, vascular/endothelial and skin components [5]. WJ-MSCs isolated from goats have been demonstrated to accelerate wound closure in animals from the same species, while minimizing granulation tissue and inflammation [6]. Human WJ-MSCs decrease 
lung [7], kidney [8] and liver [9] fibrosis, and have been shown to be able to differentiate into sweat gland-like cells and may therefore promote skin regeneration [10]. WJMSCs secrete proangiogenic and wound healing promoting factors, such as transforming growth factor beta (TGF- $\beta$ ), vascular endothelial growth factor (VEGF), platelet-derived growth factor, insulin-like growth factor-I, interleukin (IL)-6 and IL-8, among others [11]. Paracrine effects appear to be responsible for the wound healing promoting effects of WJ-MSCs, at least in mice [12]. However, to date, there are no reports regarding the use of human WJ-MSCs in human skin wounds.

The novelty of this study lies in the use of a promising stem cell type, the WJ-MSC, which has yet not been studied in the context of human skin, and investigating the in vitro effect on human skin fibroblasts, as a means to develop a new therapeutic strategy to aid in wound healing. Specifically, the aim of this study is to analyze the wound healing effects of human WJ-MSC paracrine signaling on human normal skin fibroblasts in vitro, and to examine the application of WJ-MSC conditioned medium (CM) into an in vivo mouse wound healing model.

\section{Materials and methods}

\section{Tissue sources}

Normal skin was obtained from healthy donors undergoing plastic surgery procedures (mostly dermolipectomies), both men and women, excluding pregnant females. Umbilical cords were obtained from pregnant women after planned delivery through caesarian section. Tissues were obtained at the Department of Plastic Surgery and the Department of Obstetrics and Gynecology, respectively, at Sunnybrook Health Sciences Centre, University of Toronto, Toronto, Ontario, Canada. Tissue specimens were collected following the Declaration of Helsinki Principles, following Toronto Academic Health Sciences Network (TAHSN) and University of Toronto-affiliated Sunnybrook Research Institute and Sunnybrook Health Sciences Centre Institutional Ethics Review Board approval, and after getting patient signed informed consent.

\section{Cell culture}

Primary human normal skin fibroblasts and human WJMSCs were obtained from skin tissue samples and umbilical cords, respectively. Skin was dissected to remove any underlying fat, cut into small explant pieces of 2 to $4 \mathrm{~mm}$, and cultured in small dishes. WJ-MSCs were isolated from umbilical cords by gentle dissection of previous sectioned small cord pieces, discarding the outer or epithelial layer, according to earlier described methods [13]. Explants and MSCs were further subcultured in Petri dishes at a density of 3,200 cells $/ \mathrm{cm}^{2}$ for 7 days before further passaging. When fibroblasts and/or WJ-MSCs reached $70 \%$ confluence, usually within 1 week, they were trypsinized with $0.05 \%$ trypsin $/ 0.025 \%$ ethylenediamine tetraacetic acid $\mathrm{v} / \mathrm{v}$ in preparation for subculture. Fibroblasts were subcultured in $75 \mathrm{~cm}^{2}$ tissue culture flasks at a density of 4,500 cells $/ \mathrm{cm}^{2}$. Tissue culture plasticware were purchased from BD Falcon ${ }^{\mathrm{mm}}$ (Bedford, MA, USA), and all tissue culture media and supplements were obtained from Wisent Inc. (St-Jean-Baptiste, QC, Canada), unless otherwise stated. Fibroblast culture medium consisted of highglucose Dulbecco's modified Eagle's medium (DMEM) supplemented with $10 \%$ fetal bovine serum (FBS) and 1\% antibiotic-antimycotic solution. WJ-MSC culture medium consisted of CMRL (Gibco, Carlsbad, CA, USA) with 10\% FBS, $2 \%$ antibiotic-antimycotic solution and $1 \% \mathrm{~L}$-glutamine. Media were changed every 48 hours. Collected tissues and cells were cultured at $37^{\circ} \mathrm{C}$ in a humidified atmosphere with $5 \%$ carbon dioxide.

\section{Characterization of human Wharton's jelly-derived MSCs}

The cells isolated from the Wharton's jelly of the umbilical cord were examined to confirm their MSC characteristics. Flow cytometry for MSC cell surface markers $\left(\mathrm{CD}^{\circ}{ }^{+}, \mathrm{CD}_{3}{ }^{+}, \mathrm{CD} 105^{+}, \mathrm{CD} 45^{-}, \mathrm{CD} 14^{-}, \mathrm{CD} 34^{-}, \mathrm{CD} 19^{-}\right.$ and HLA-DR ${ }^{-}$) was performed (Additional file 1). Cells were differentiated into the three main mesenchymal lineages - adipogenic, osteogenic and chondrogenic (Additional file 1). For adipogenic differentiation, cells were seeded at a density of 3,000 cells $/ \mathrm{cm}^{2}$ in 24-well plates (BD) with low-glucose DMEM (Wisent Inc., St-JeanBaptiste, QC, Canada) medium supplemented with $10 \%$ FBS, $1 \%$ antibiotic-antimycotic solution, $1 \mathrm{mM}$ 3-isobutyl1-methylxanthine (Sigma-Aldrich, Saint Louis, MO, USA), $10 \mu \mathrm{g} / \mathrm{ml}$ insulin (SAFC, Saint Louis, MO, USA), $60 \mu \mathrm{M}$ indomethacin (Sigma-Aldrich), and $1 \mu \mathrm{M}$ dexamethasone (Sigma-Aldrich). Cultures of cells in low-glucose DMEM medium supplemented with 10\% FBS served as a negative control. Lipid accumulation was identified by oil red $\mathrm{O}$ staining: $0.3 \mathrm{~g}$ oil red $\mathrm{O}$ (Sigma-Aldrich) dissolved in $100 \mathrm{ml}$ isopropanol (Sigma-Aldrich), diluted to $60 \%$ with distilled water.

For osteogenic differentiation, cells were also seeded at a density of 3,000 cells $/ \mathrm{cm}^{2}$ in 24-well plates with low-glucose DMEM supplemented with 10\% FBS, 1\% antibiotic-antimycotic solution, $0.05 \mathrm{mM}$ ascorbic acid-2phosphate (Wako Pure Chemicals Industry Ltd, Osaka, Japan), $10 \mathrm{mM} \beta$-glycerophosphate (Sigma-Aldrich), and $100 \mathrm{nM}$ dexamethasone (Sigma-Aldrich). Alizarin red staining (Sigma-Aldrich) was used to identify osteogenic cells ( $2 \mathrm{~g}$ alizarin red dissolved in $100 \mathrm{ml}$ distilled water). For chondrogenic differentiation, cells were seeded in $15 \mathrm{ml}$ polypropylene tubes $\left(2 \times 10^{5}\right.$ cells per tube; BD Falcon, Bedford, MA, USA) with low-glucose DMEM supplemented with $10 \%$ FBS, $1 \%$ antibiotic-antimycotic solution, $1 \mathrm{mM}$ sodium pyruvate (Sigma-Aldrich), 
0.1 mM ascorbic acid-2-phosphate (Wako Pure Chemicals Industry Ltd), 1\% insulin-transferrin-selenium (Cellgro, Manassas, VA, USA), 100 nM dexamethasone (SigmaAldrich), and $10 \mathrm{ng} / \mathrm{ml}$ TGF- $\beta 3$ (Shenandoah Biotechnology, Inc., Warwick, PA, USA). Chondrocyte pellets were identified with Safranin O staining: $0.1 \mathrm{~g}$ Safranin O (Sigma-Aldrich), dissolved in $100 \mathrm{ml}$ distilled water.

\section{Human normal fibroblasts and Wharton's jelly-derived MSC one-way paracrine signaling indirect co-culture}

First, primary human fibroblasts were seeded into sixwell plates (Grenier-Bio-One Cellstar, Frieckenhausen, Germany) at a density of 22,000 cells $/ \mathrm{cm}^{2}$ with DMEM. To design a one-way indirect or paracrine co-culture system between human normal skin fibroblasts and WJMSCs, the WJ-MSCs were separately seeded at the same cell density in the upper three wells of a six-well plate with CMRL media; the lower three wells of the same six-well plate were filled with CMRL medium alone. When refreshing the media (once every other day), the six-well plate containing the fibroblasts was filled with the medium from the WJ-MSC six-well plate: the three upper wells (treatment wells) with the WJ-MSC-CM, and the three lower wells (control wells) with the CMRL media alone. On day 5 of culture, the amount of FBS in the medium was reduced from 10 to $2 \%$, to avoid TGF$\beta 1$ false measurements. On day 7 , total RNA extraction was started for further gene expression studies. Experiments were performed with low-passage cells (less than passage 5) and in triplicate (unless otherwise stated), on one set of cells from different patients.

\section{RNA isolation and real-time quantitative polymerase chain reaction}

For RNA isolation, cells were lysed using TRIzol reagent (Invitrogen, Carlsbad, CA, USA), and the RNeasy MicroKit was used (Qiagen, Inc., Valencia, CA, USA) according to the manufacturer's instructions. The total RNA yield was determined using a NanoDrop-2000 spectrophotometer (ThermoScientific, Waltham, MA, USA). cDNA was synthesized in a thermocycler (AB Applied Biosystems, Foster City, CA, USA), after mixing $10 \mu \mathrm{g}$ RNA and a master mix prepared with the high-capacity cDNA synthesis reverse transcription kit (AB Applied Biosystems). Real-time polymerase chain reaction (PCR) was conducted using $\mathrm{SYBR}^{\bullet}$ Green PCR Master Mix (Applied Biosystems) to relatively quantify the mRNA transcript products of the following genes of interest: TGF- $\beta 1, T G F-\beta 2, T G F-\beta 3$, plasminogen activator inhibitor-1, connective tissue growth factor, fibroblast growth factor-2, hypoxia-inducible factor-1 $\alpha$, VEGF, collagen I, collagen III and decorin. $18 \mathrm{~S}$ was used as housekeeping gene. The primer sequences of the above genes used are listed in Additional file 2. Amplification and analysis of cDNA fragments were carried out using the StepOnePlus RT-PCR System (AB Applied Biosystems).

Relative gene expression was measured as the cycle threshold and was normalized with individual housekeeping gene control cycle threshold values. Quantitative PCR was loaded in duplicate, and cycle threshold values from triplicates of the same treatment group sample were averaged. The $\Delta \Delta C$ t method was used to report quantitative PCR results.

\section{Proliferation assay: Ki67 antigen staining}

Normal skin fibroblasts and WJ-MSCs were seeded in different eight-chamber culture slides at a cell density of 715 cells $/ \mathrm{cm}^{2}$ and cultured for 7 days, following methods described above. Briefly, the media from the wells containing WJ-MSC (WJ-MSC-CM) were used to fill the wells from the normal skin fibroblasts from the treated group every other day, whereas wells of the control group were filled with WJ-MSC nonconditioned media.

\section{Immunofluorescence}

Cells were washed with phosphate-buffered saline (PBS) and fixed for 15 minutes in 4\% paraformaldehyde (Alfa Aesar, Karlsruhe, Germany). Fixed cells were washed in PBS and permeabilized for 10 minutes with PBS/0.5\% Triton X-100 solution. After another washing step, cells were blocked for 30 minutes with $1 \%$ bovine serum albumin in PBS/0.5\% Triton X-100. A monoclonal mouse anti-human Ki67 (1:100, clone MIB-1; Dako, Markham, ON, Canada) primary antibody was added and incubated overnight at $4^{\circ} \mathrm{C}$. After washing with PBS, the secondary antibody was added in $1 \%$ bovine serum albumin in PBS/ $0.5 \%$ Triton X-100 and incubated for 1 hour at room temperature in the dark (Alexa Fluor 488 donkey antimouse, 1:500; Life Technologies, Eugene, OR, USA). After three final washes with PBS, slides were mounted with Vectashield mounting medium with 4',6-diamidino2-phenylindole (DAPI; Vector Laboratories, Burlingame, CA, USA). Cells were examined and photographed using an Apotome Axiovert fluorescent imaging system at $10 \times$ magnification (Zeiss, Oberkochen, Germany). Three images were taken per well and two wells were imaged per treatment. Quantification was performed by counting the number of Ki67-positive cells in the high-power field as well as the total number of DAPI-positive nuclei. Data are presented as means with 95\% confidence intervals of duplicate measurements for three different normal skin samples.

\section{Terminal transferase TdT-mediated dUTP biotin end-labeling apoptosis assay}

Normal fibroblasts cultured with WJ-MSC-CM and with nonconditioned medium were seeded into eight-chamber 
culture slides at a cell density of 3,000 cells $/ \mathrm{cm}^{2}$ for half a week. The terminal transferase TdT-mediated dUTP biotin end-labeling apoptosis kit (Promega, Fitchburg, WI, USA) was used as per the manufacturer's instructions.

Briefly, cells were fixed in $4 \%$ paraformaldehyde for 25 minutes at $4^{\circ} \mathrm{C}$ and washed with PBS. They were permeabilized with $0.25 \%$ Triton X-100 in PBS for 5 minutes, and then washed with PBS. Cells were incubated with equilibration buffer for 10 minutes, followed by labeling with terminal deoxynucleotidyl transferase reaction mix ( $10 \%$ nucleotide mix, $0.02 \% \mathrm{rTdT}$ enzyme in equilibration buffer) for 1 hour at $37^{\circ} \mathrm{C}$ in humidified chamber. Cells were immersed in $2 \times$ SSC for 15 minutes to stop the reaction, followed by washes with PBS. Slides were mounted with Vectashield mounting medium with DAPI (H-1200; Vector Laboratories). Images were taken on an Apotome Axiovert fluorescent imaging system at 10× magnification (Zeiss); three images were taken per well. Quantification was performed by counting the number of terminal transferase TdT-mediated dUTP biotin end-labeling-positive and DAPI-positive nuclei, or apoptotic and alive cells, respectively.

\section{Cell migration study: scratch wound assay}

Normal skin fibroblasts were seeded in four-chamber culture slides at a cell density of 1,000 cells $/ \mathrm{cm}^{2}$, with WJ-MSC-CM (or non-conditioned medium as control) for 48 hours. Two scratches were performed with a $200 \mu$ pipette tip. After 24 hours, cells were fixed with $4 \%$ paraformaldehyde. The staining protocol followed the same procedure as the aforementioned proliferation assay. Briefly, cells were incubated with phalloidin antibody conjugated to fluorescein isothiocyanate (1:30; Invitrogen, Eugene, OR, USA) in blocking solution for 1 hour. Cells were washed three times with PBS and mounted with Vectashield mounting medium with DAPI. Images were taken on laser scanning META 510 confocal microscope (Zeiss) with $5 \times$ magnification. Three images were taken per scratch. Quantification was performed using Image J software (National Institutes of Health, Bethesda, MD, USA). A set area with a height of $0.5 \mathrm{~mm}$ and a width spanning the high-power field was placed in the center of the scratch, and the cells within this area were counted as the cells in scratch zone.

\section{In vivo wound healing model}

Eight BALB/c mice (13 weeks old, male, body weight 28 to $34 \mathrm{~g}$ ) were obtained from Jackson Laboratory under the guidelines of the Sunnybrook Research Institute and Sunnybrook Health Sciences Animal Policy and Welfare Committee of the University of Toronto. Animal procedures were reviewed and approved by Sunnybrook Research Institute and Sunnybrook Health Sciences Centre at University of Toronto animal care and use committee.
Animals were anesthetized and back cutaneous hair was removed by electrical shaving under anesthesia as stated in the Animal Protocol. Two pairs of $4 \mathrm{~mm}$ diameter full-thickness skin excisional wounds were created on each side of the midline. The animals were randomly divided into two groups: treatment (WJ-MSC-CM and Matrigel; BD Biosciences, San Jose, CA, USA) and sham (nonconditioned medium and Matrigel). Matrigel was of high concentration and was applied dropwise in liquid form and then allowed to gel. Each wound topically received $100 \mu \mathrm{l}$ treatment or sham mix.

\section{Wound analysis}

Wound measurements were taken and wound closure was examined in a timely manner on days 1,3 and 7 . Wounds with a complete re-epithelialization were considered healed wounds.

Mice were sacrificed at day 7 , when skin biopsies including the wound/scar and $2 \mathrm{~mm}$ of satellite skin were harvested for further histologic analysis. Twenty-four hours before sacking, animals received an intraperitoneal injection of bromodeoxyuridine (BrdU) (Calbiochem, San Diego, CA, USA).

\section{Histologic examination}

Tissue specimens were fixed in $10 \%$ buffered formalin overnight at room temperature, preserved in $70 \%$ ethanol and embedded in paraffin. Specimens were cut into $5 \mu \mathrm{m}$ sections. Tissue specimens were cut simultaneously at different sites, the center or midline and both sides, eliciting a cross-section through the whole wound and satellite area. A serial section of the scar or healing wound was performed. The largest wound diameter or central wound section was stained for trichrome staining. Trichrome reagents were from EMS (Hatfield, PA, USA) unless otherwise stated. Briefly, paraffin-embedded slides were deparaffinized with citrosol, followed by rehydration through 100\%, 95\%, 70\% and 50\% ethanol to water. Slides were placed in Bouin's solution (26367-01; EMS, Hatfield, PA, USA) overnight at room temperature and washed next. Hematoxylin stain (HHS16; Sigma, Saint Louis, MO, USA) and Biebrich scarlet-acid fuchsin solution were applied sequentially for 10 minutes. Washes were performed after each stain addition. Slides were differentiated in phosphomolybdic-tungstic acid for 15 minutes, and were transferred to aniline blue for 5 minutes. They were next rinsed and differentiated in $1 \%$ acetic acid for 2 minutes. Slides were dehydrated through 95\% ethanol and absolute ethanol followed by clearing in citrosol. Slides were mounted with SHUR/ Mount xylene-based liquid mounting media (Triangle Biomedical Sciences, Durham, NC, USA). Images were acquired using a Zeiss Axiovert 200 light microscope at 10x and 40x magnification. Quantification was carried out 
using merged $10 \times$ images to measure the wound bed and satellite area.

For immunohistochemistry staining, paraffin-embedded skin tissue slides were deparaffinized with xylene followed by rehydration. Antigen decloaker (1×; Biocare Medical, Concord, CA, USA) was added to the slides in a preheated decloaking chamber for 4 minutes at $110^{\circ} \mathrm{C}$. For $\mathrm{BrdU}$ staining, samples were denatured with $1.5 \mathrm{~N} \mathrm{HCl}$ for 30 minutes at $37^{\circ} \mathrm{C}$ and neutralized with $0.1 \mathrm{M}$ borate buffered twice for 5 minutes. Samples were blocked with $3 \% \mathrm{H}_{2} \mathrm{O}_{2}$ for 10 minutes, and then washed with washing buffer $(0.05 \mathrm{M}$ Tris- $\mathrm{HCl}, 0.15 \mathrm{M} \mathrm{NaCl}, 0.05 \%$ Tween 20 in deionized water). The primary antibody (mouse monoclonal anti-BrdU, 1:200; Cell Signaling, Beverly, MA, USA) was diluted in PBS and incubated at room temperature for 1 hour. Slides were then incubated for 15 minutes first with MACH3 mouse probe (Biocare Medical), and secondly with MACH3 rabbit or mouse horseradish peroxidase polymer, with before and after washes. The betazoid diaminobenzidine chromogen kit (Biocare Medical) was mixed and added for 5 minutes or until brown stain was noticeable. The reaction was terminated with running water. Nuclear staining was carried out with hematoxylin for 30 seconds, followed by differentiation with three dips in $1.5 \%$ acid alcohol and bluing in $0.1 \%$ sodium bicarbonate for 10 seconds. Sections were dehydrated through 95\% and absolute ethanol to citrosol and mounted with SHUR/Mount as described previously. Images were acquired using a Zeiss Axiovert 200 light microscope at 10x magnification to image the whole section followed by $40 \times$ magnification to further focus on the wound margins and the wound center. The higher magnification images of BrdU staining were quantified by counting using ImageJ software, and normalized to the number of cells in the high-power field.

\section{Statistical analysis}

The statistical comparisons between the groups were performed using an unpaired Student's $t$ test with GraphPad Prism software (GraphPad, LaJolla, CA, USA). Two-tailed $P \leq 0.05$ was considered significant. Data were graphically expressed as the mean of the target group \pm the standard error of the mean or $95 \%$ confidence interval.

\section{Results}

Wharton's jelly-derived MSCs enhance the expression of wound healing genes by paracrine signaling

Human WJ-MSCs upregulated the mRNA transcript expression of TGF- $\beta 2$, hypoxia-inducible factor-1 $\alpha$, and plasminogen activator inhibitor-1 genes $(P \leq 0.05)$ in normal skin fibroblasts in our culture conditions. Other genes involved in re-epithelialization, neovascularization and/or remodeling - including VEGF, fibroblast growth factor-2, connective tissue growth factor, collagen I and collagen
III - were not changed. Decorin and TGF- $\beta 3$ also remained unaffected (Figure 1).

\section{Normal skin fibroblasts proliferate faster when treated} with Wharton's jelly-derived MSC conditioned medium In our culture conditions, WJ-MSC-CM accelerated normal skin fibroblasts proliferation $(P \leq 0.001)$, as measured by a Ki67 proliferation assay (Figure 2A,B,C).

\section{Wharton's jelly-derived MSC conditioned medium does not induce apoptosis in normal skin fibroblasts}

We did not find any significant modulation in the number of apoptotic fibroblasts treated with WJ-MSC-CM using a terminal transferase TdT-mediated dUTP biotin end-labeling assay, suggesting that WJ-MSC-CM does not appear to affect normal skin fibroblast apoptosis under our culture conditions (Figure 2D). An increased apoptosis rate could imply a delayed wound healing process, whereas a much decreased apoptosis rate could imply enhanced risk of keloid scars.

\section{Wharton's jelly-derived MSC conditioned medium promotes normal skin fibroblast migration and wound closure}

To further examine whether the enhanced proliferation of fibroblasts treated with WJ-MSC-CM may also promote wound closure, a wound scratch assay was performed.

In our culture conditions, WJ-MSC-CM-treated normal skin fibroblasts coapted wound borders faster than normal skin fibroblasts from the control group (that is, those treated with WJ-MSC nonconditioned medium), suggesting that WJ-MSC-CM enhanced fibroblast migration. This observation is important, because cell migration is an essential step during wound healing. This fact highlights that healing promotion is partly due to faster migration, and consequently acceleration of wound closure, in human normal skin fibroblasts (Figure 3A,B).

\section{Wharton's jelly-derived MSC conditioned medium pro- motes wound healing and repair in a mouse model} We next examined whether the in vitro wound healing promoting observed effects with WJ-MSC-CM might be translated into an in vivo wound healing model. BALB-c mice with WJ-MSC-CM-treated wounds showed enhanced wound healing rates compared with the control mice $(P \leq 0.05)$ (Figure $4 \mathrm{~A}, \mathrm{~B}$, quantified in $4 \mathrm{C})$. Increased and complete re-epithelialization, higher cellularity in newly formed granulation tissue, and less random and more organized extracellular matrix were observed in the WJ-MSC-CM-treated wounds, suggesting that WJ-MSCCM promoted wound healing and repair in vivo in mice.

To delineate the pro-proliferative effect of WJ-MSC$\mathrm{CM}$ in vivo, one dose of BrdU was injected intraperitoneally. Both a higher number of cells and a higher amount of 


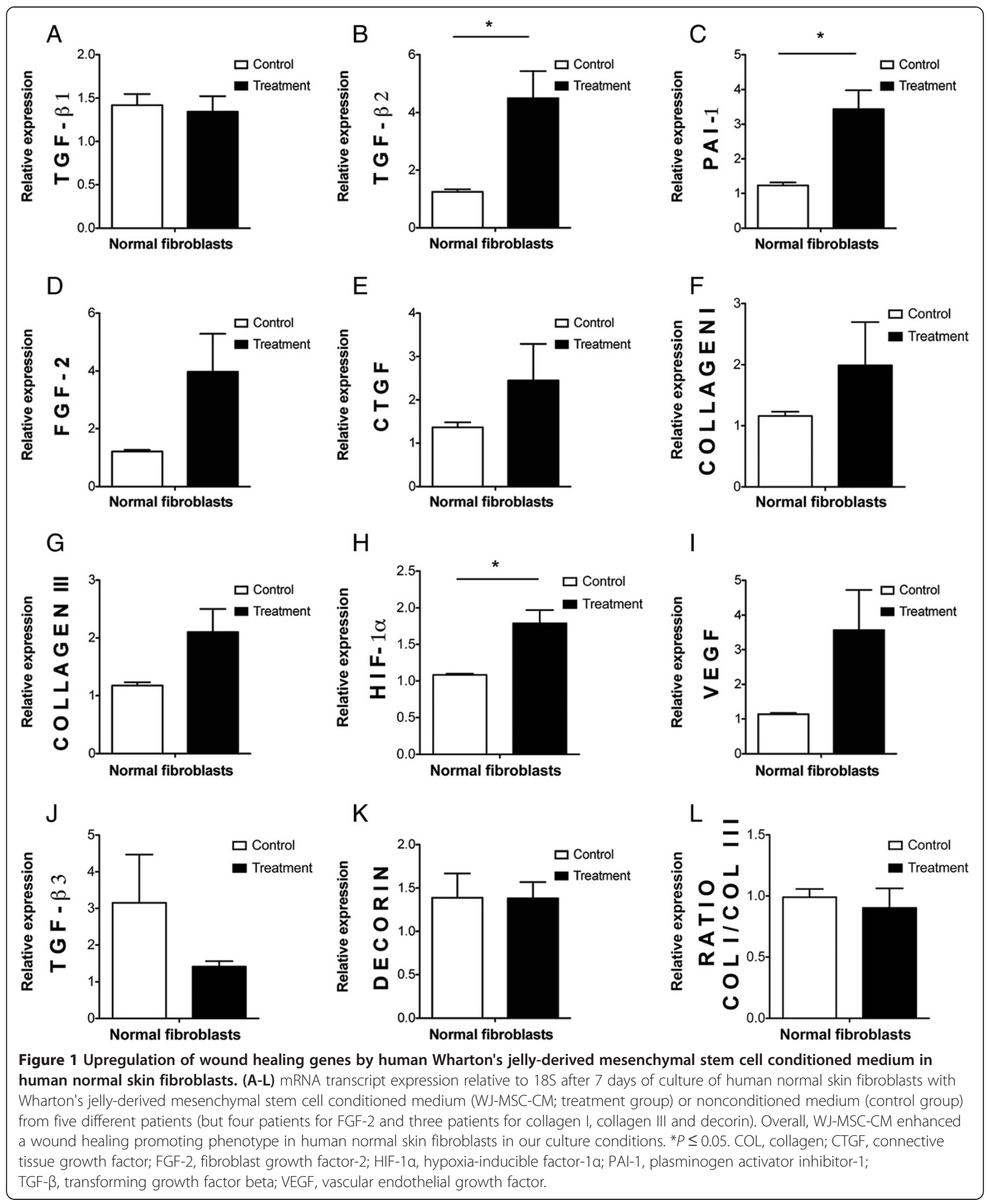

proliferative cells were found in the WJ-MSC-CM-treated wounds ( $P \leq 0.05$ and $P \leq 0.01$, respectively) (Figure $5 \mathrm{E}, \mathrm{F})$. A microscopic wound cross-section showed a higher number of positive proliferating nuclei (black arrows, BrdU-positive cells $)(P \leq 0.01)$ in the WJ-MSC-CM-treated wounds (Figure 5D) compared with the control wounds 


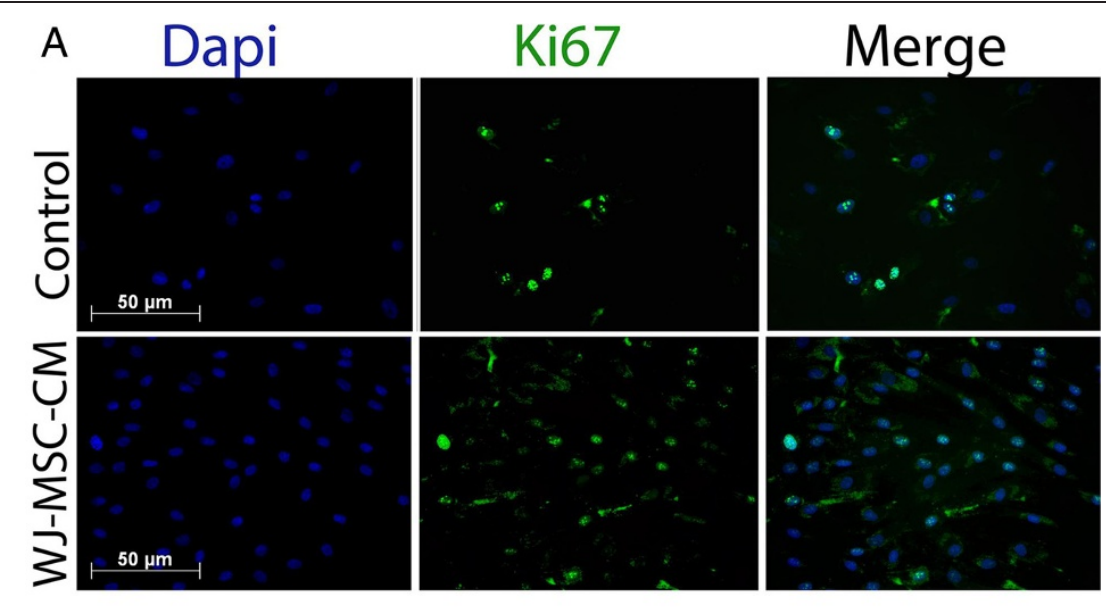

B

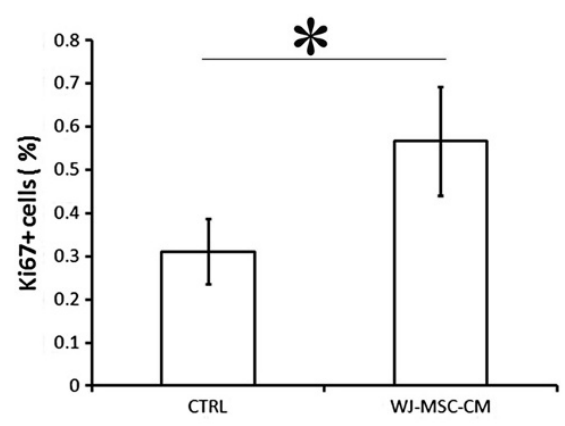

C

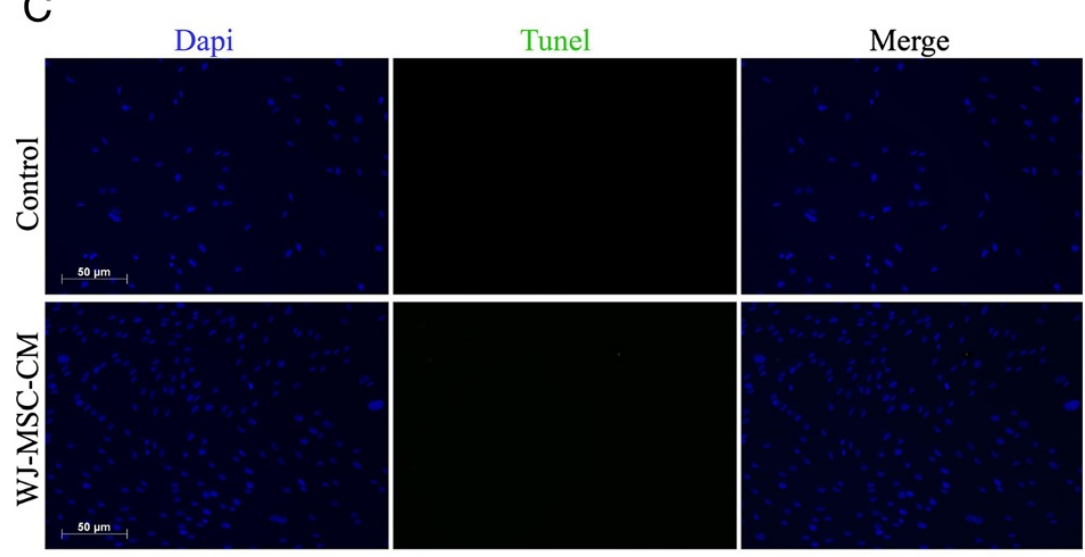

D

E
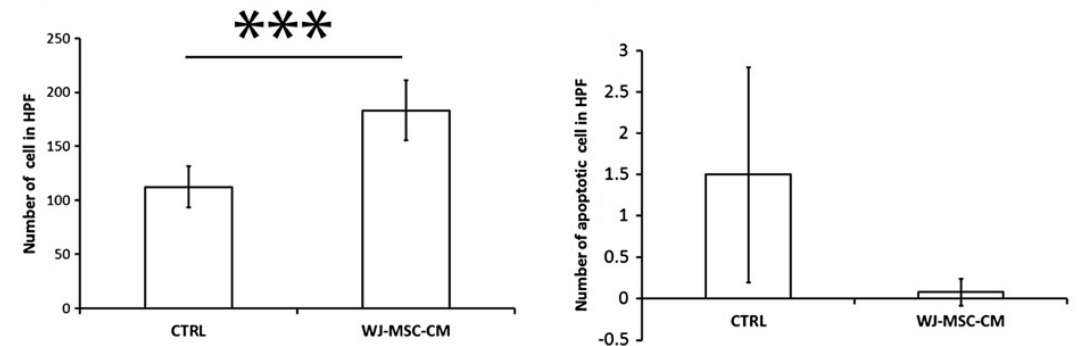

Figure 2 (See legend on next page.) 
(See figure on previous page.)

Figure $\mathbf{2}$ Wharton's jelly-derived mesenchymal stem cell conditioned medium increases normal skin fibroblast proliferation, but does not affect apoptosis. Cell proliferation was examined using Ki67 staining. (A) Wharton's jelly-derived mesenchymal stem cell conditioned medium (WJ-MSC-CM)-treated normal fibroblasts showed enhanced proliferative rates compared with the control group (quantified in (B)). (C) Terminal transferase TdT-mediated dUTP biotin end-labeling (TUNEL) staining of WJ-MSC-CM-treated human normal skin fibroblasts versus control (non-WJ-MSC-CM-treated) normal skin fibroblasts showed no significant difference in induction of apoptosis (quantified in (E)). Note that the total number of viable cells was significantly higher in the WJ-MSC-CM-treated cells compared with the non-WJ-MSC-CM-treated cells (D). $n=3$ samples per group. ${ }^{*} P \leq 0.05,{ }^{* * *} P \leq 0.001$. DAPI, 4',6-diamidino-2-phenylindole.

(Figure 5C), as well as general increased cellularity, matrix remodeling and overall wound repair. Higher magnification images corresponding to the aforementioned detailed micrographs of both control and treated wounds were shown in Figure 5A and 5B, respectively (Additional file 3 supports and complements Figure 5).

\section{Discussion}

The results of this study suggest that WJ-MSCs enhance wound healing and normal wound repair by paracrine signaling mechanisms. Under our culture conditions, human WJ-MSC-CM upregulated the gene expression of wound healing factors in human normal skin fibroblasts, and promoted fibroblast proliferation and migration to coapt wound borders in vitro. Accordingly, WJ-MSC-CM accelerated the re-epithelialization rate and promoted wound repair in vivo in a full-thickness excisional mouse wound healing model.

Cutaneous wound repair is a complex orchestrated process that is activated upon injury and includes the

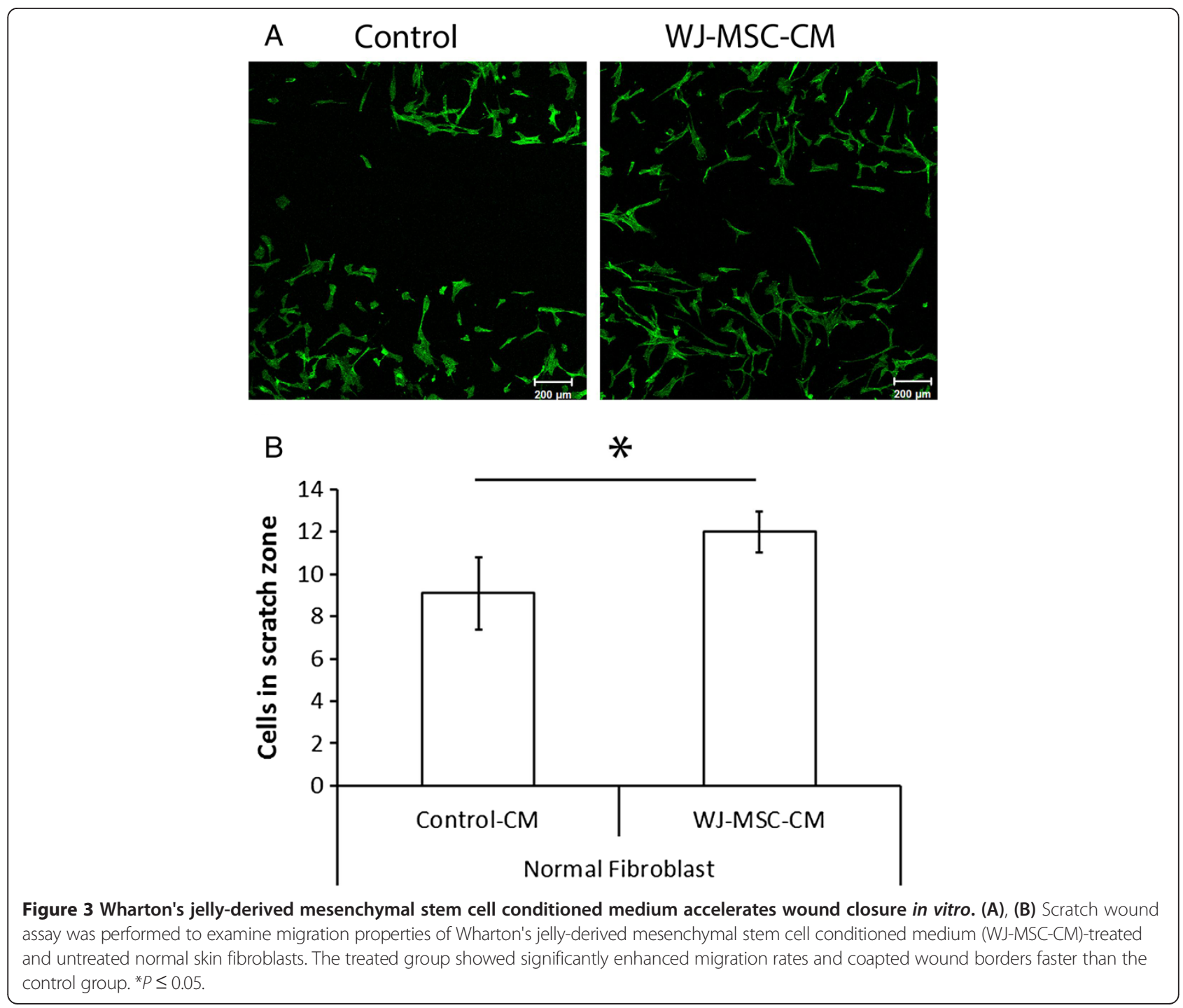



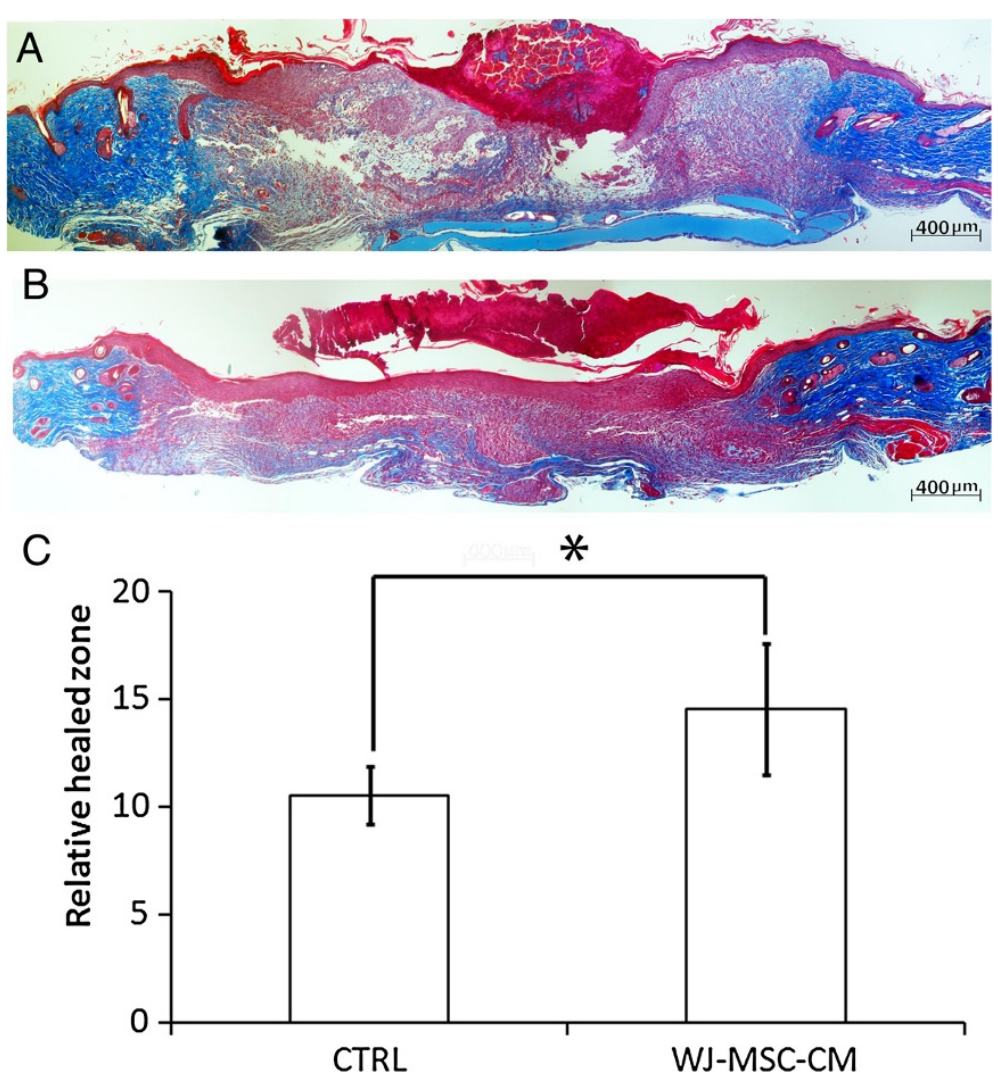

Figure 4 Wharton's jelly-derived mesenchymal stem cell conditioned medium enhances wound healing in an in vivo mouse model. A murine wound healing model was used, and animals were wounded and treated as described previously. Images corresponded to histological sections of wounds and the satellite donut area (10X) from BALB-c mice, after 1 week of full-thickness excisional skin wounding and reconstruction with Wharton's jelly-derived mesenchymal stem cell conditioned medium (WJ-MSC-CM) and vehicle (Matrigel; BD Biosciences, San Jose, CA, USA) (B), or vehicle alone (A). Photomicrographs were taken after Masson's Trichrome staining. BALB-c mice WJ-MSC-CM-treated wounds showed enhanced wound healing rates compared to the control mice $(P \leq 0.05)(\mathbf{C}) .{ }^{*} P \leq 0.05$. Error bars represent the $95 \%$ confidence interval.

multicellular overlapping and coordinated phases of inflammation, angiogenesis and formation of granulation tissue, re-epithelialization, and fibroproliferation or matrix formation and remodeling [14,15]. Decreased proinflammatory cytokines, compromised neovascularization and/ or impairment in leukocyte recruitment might disturb and delay wound healing [16]. Despite the current use and availability of a wide array of wound dressings, ointments and devices, wound healing still remains a clinical challenge, especially in older patients, diabetic patients, heavy smokers or burned patients [17-21]. There is therefore a need for new strategies to promote or at least coadjuvantly help in wound healing and repair.

Skin MSCs have been reported to populate the normal skin niche, remain quiescent and become active after injury, aiding in wound closure $[14,15]$. MSC paracrine signaling has been suggested to be the main underlying mechanism for MSCs enhanced wound repair effects $[2,22,23]$. BM-MSCs have been reported to promote wound healing, but their isolation requires an invasive and artificial method [24,25]. Harvesting adipose-derived stem cells also requires a surgical procedure. Subsequently, we focused on a more advantageous MSC source, the umbilical cord-derived Wharton's jelly $[5,25,26]$. The harvest of WJ-MSCs is not painful or invasive, as the cells are isolated naturally with no extra surgery and are dissected from discarded umbilical cords after birth. Indeed, caprine WJMSCs have already been shown to promote wound repair with minimal scarring [6], but to our knowledge there are no reports of human WJ-MSC treatment on human skin wounds. WJ-MSCs represent a very efficient stem cell source with reported immunoprivileged, anticancer and antifibrotic characteristics in animal models [25-28]. Due to their reported immunoprivileged properties and universal and ever-lasting availability [5], WJ-MSC allotransplantion as an off-the-shelf therapy may represent an appropriate treatment strategy in the already compromised patients who suffer of recalcitrant cutaneous wounds [14].

This study therefore aimed to examine the effects of human WJ-MSC paracrine signaling on human normal skin fibroblasts. In our culture conditions, human WJMSCs enhanced the expression of some wound healing 


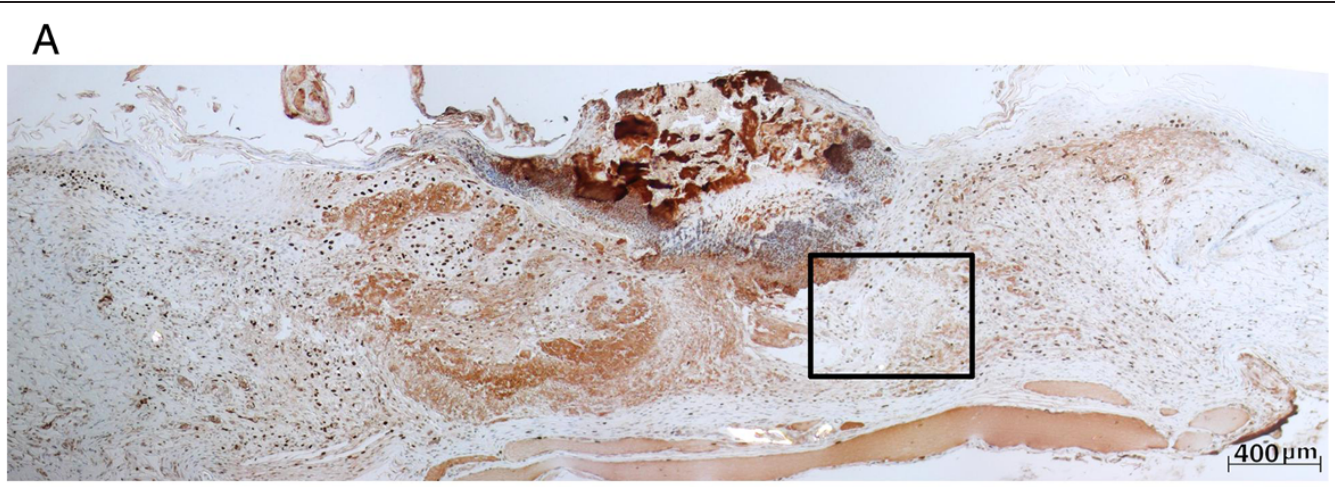

\section{B}

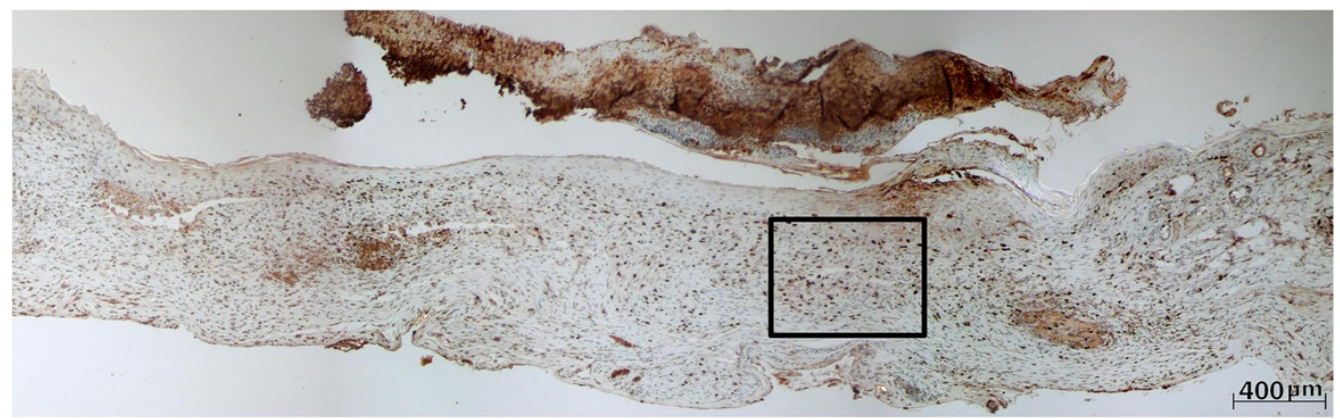

C

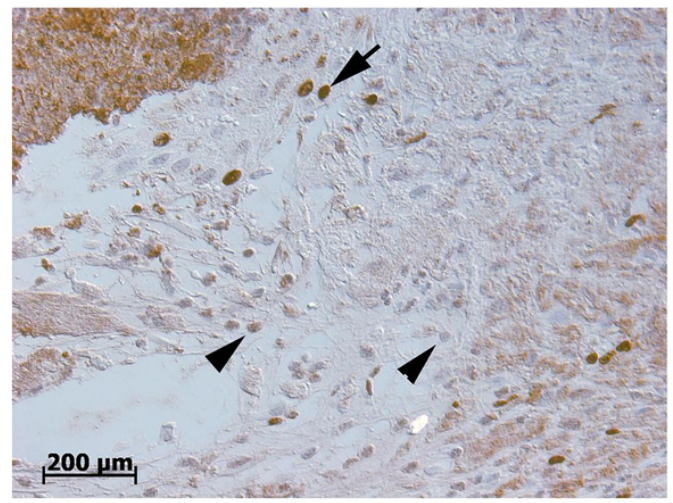

E

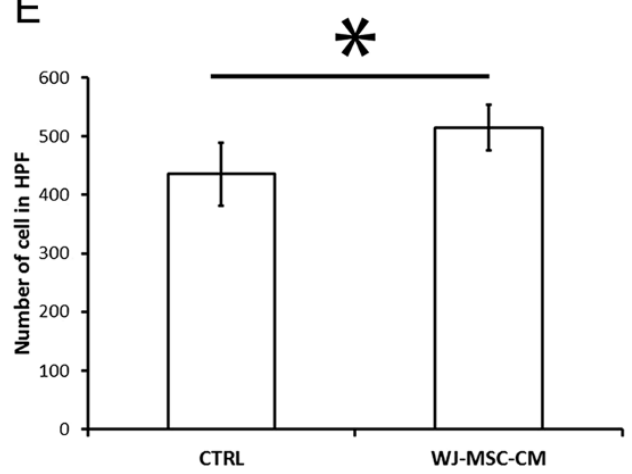

D

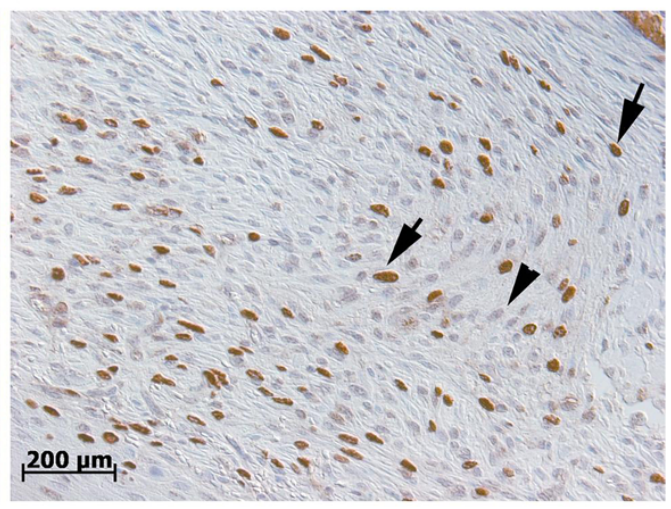

$\mathrm{F}$

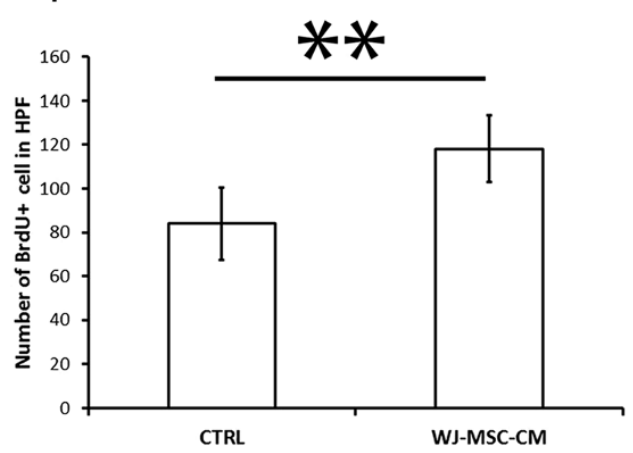

Figure $\mathbf{5}$ (See legend on next page.) 


\section{(See figure on previous page.)}

Figure 5 Wharton's jelly-derived mesenchymal stem cell conditioned medium promotes cell proliferation in an in vivo mouse wound healing model. A BALB-c mouse wound healing model was used and animals were wounded and treated as described previously. Animals received one dose of bromodeoxyuridine $(\mathrm{BrdU})$ intraperitoneally 24 hours before harvesting of wounds. Four animals were included in each group, and four wounds were performed per animal (total of 16 wounds in each group). Cutaneous tissue specimens were stained for BrdU in both groups, control (A) and treatment (B). Enhanced magnification (40X) of the above microscopic images were included for nonconditioned medium-treated (C) and Wharton's jelly-derived mesenchymal stem cell conditioned medium (WJ-MSC-CM)-treated normal skin fibroblasts (D) to examine in further detail the increase in cell number or stained nuclei (black arrows, BrdU-positive cells) in the WJ-MSC-CM-treated wounds, compared with controls. This denoted that WJ-MSC-CM stimulated cell proliferation in vivo (E, F). Together, these results suggest that WJ-MSC promoted wound healing and repair by one-way paracrine signaling in an in vivo preclinical model. ${ }^{*} P \leq 0.05$, ${ }^{* *} P \leq 0.01$. Error bars represent the 95\% confidence interval. Arrows, BrdU-positive nuclei; arrowheads, BrdU-negative nuclei. HPF, high-power field.

promoting genes, including re-epithelialization, neovascularization and fibroproliferation inducing genes, in human normal skin fibroblasts. Besides altering skin fibroblast gene expression, MSC signaling has also been shown to positively regulate cell survival, proliferation and migration [23,29]. Under our culture conditions, although apoptotic changes were not found in skin fibroblasts treated with WJ-MSC-CM, significantly enhanced proliferation and migration were observed. This is important, as cellular dynamics and cell migration constitute an essential step during cutaneous healing. WJ-MSC-CM accelerated wound closure both in vitro and in an in vivo mouse wound healing model, suggesting that human WJMSC-CM may promote wound repair.

Other researchers have also reported enhanced wound healing rates in mouse models with other human MSC sources, such as BM-MSCs [23,30] and umbilical cord blood-derived MSCs [31]. Blood of the umbilical cord has been reported to yield fewer MSCs than the cord itself [31], however, and Wharton's jelly may therefore emerge as a more appropriate stem cell source. To the best of our knowledge, no published studies regarding the use of human WJ-MSCs in human wounds have been reported, so this work is novel, and could prompt us to test whether the same results would be encountered first in a more appropriate but more costly experimental model, such as the red duroc pig. Secondly, if the results appeared to be promising, the next step would be to translate them into phase I or phase II clinical trials for further evaluation and development. Indeed, pilot clinical studies have so far indicated that MSCs in general are safe in vivo, and they currently represent the most widely used stem cells in the clinical setting [26]. Accordingly, in the particular case of WJ-MSCs, 15 diabetic patients received systemically WJ-MSCs with no documented relevant safety concerns [32]. Furthermore, many MSC clinical applications to manage intestinal fistulae, acute artery ischemic disease in diabetic patients, and periodontal defects have been reported, among others [26]. Anecdotally, one case report about autologous BMMSCs used to help to heal a scapular recalcitrant wound in a Russian burned patient has also been published [33], but no reports regarding the use of WJ-MSCs have yet been reported. Although WJ-MSCs secrete a more angiogenic secretome in comparison with BM-MSCs [34], the isolation of WJ-MSCs is more efficient, and WJ-MSCs have a higher capacity of proliferation and are less senescent than adipose-derived stem cells [35], proper studies comparing the wound healing abilities of different MSC sources are still lacking in the literature. On the other hand, the direct effects of human WJ-MSCs on wound healing, regeneration and repair still remain unknown. A new preclinical and clinical research arena investigating the potential of WJ-MSCs in wounds and wound healing has just been born and may hold promise for future medical therapies.

\section{Conclusions}

Human WJ-MSCs promoted wound healing by paracrine signaling in our culture conditions in vitro, and in an in vivo preclinical animal model. If the reported immunoprivileged character and safety of WJ-MSCs observed in experimental and first clinical models is further scientifically proven, WJ-MSCs might represent a feasible, universal and off-the-shelf technology to enhance normal wound healing to improve patient survival and quality of life.

\section{Additional files}

Additional file 1: Figure S1. Showing flow-cytometry markers and mesenchymal differentiation of human WJ-MSCs. Flow cytometry analysis of established human WJ-MSCs (successfully grown on plastic plates) showing markers used to characterize MSCs $(a, b, c, d)$. Cells were able to differentiate into osteocytes (e), chondrocytes (f) and adipocytes (g). Images shown after alizarin red (e), safranin $\mathrm{O}(\mathrm{f})$ and oil red $(\mathrm{g})$ staining, respectively.

Additional file 2: Table S1. Showing primer sequences for real-time PCR. List of SYBR ${ }^{\circledR}$ Green gene primer sequences used in real-time PCR for the present work. *TGF- $\beta$, transforming growth factor- $\beta$; CTGF, connective tissue growth factor; PAI-I, plasminogen activator inhibitor-l; HIF-1-a, hypoxia inducible factor-1-a; VEGF, vascular endothelial growth factor; FGF-2, fibroblast growth factor-2.

Additional file 3: Figure S2. Showing WJ-MSC-CM promoted cell proliferation in an in vivo wound healing model in BALB-C mice. (A) BrdU staining in the control group. (B) BrdU staining in the treatment group. (C), (D) Higher magnification (40x) of the above microscopic images were included for nonconditioned medium-treated ( $C$ is magnification of the marked area in A) and WJ-MSC-CM-treated normal skin fibroblasts (D is magnification of the marked area in B) to examine in further detail the 
increase in cell number or stained nuclei in the WJ-MSC-CM-treated wounds, compared with controls. Brown nuclei correspond to cells that incorporated BrdU into their DNA. (Supplemental figure in support of Figure 5).

\section{Abbreviations}

BM-MSC: bone marrow-derived mesenchymal stem cell; BrdU: bromodeoxyuridine; CM: conditioned medium; DAPI: 4,'6-diamidino-2phenylindole; DMEM: Dulbecco's modified Eagle's medium; FBS: fetal bovine serum; IL: interleukin; MSC: mesenchymal stem cell; PBS: phosphate-buffered saline; PCR: polymerase chain reaction; TGF- $\beta$ : transforming growth factor beta; VEGF: vascular endothelial growth factor; WJ-MSC: Wharton's jellyderived mesenchymal stem cell.

\section{Competing interests}

The authors declare that they have no competing interests or any potential conflict of interest in any of the techniques or instruments mentioned in this manuscript.

\section{Authors' contributions}

AlA participated in the conception and design, administrative support, collection and/or assembly of data, data analysis and interpretation, manuscript writing and final approval of the manuscript. SA-N contributed to the conception and design, administrative support, collection and/or assembly of data, data analysis and interpretation, manuscript writing and final approval of the manuscript. PHB participated in the conception and design, administrative support, collection and/or assembly of data, data analysis and interpretation, manuscript writing and final approval of the manuscript. MA-S contributed to administrative support, collection and/or assembly of data and final approval of the manuscript. CB provided administrative support and helped in collection and/or assembly of data and final approval of the manuscript. EH carried out provision of study material or patients, administrative support, helped in collection and/or assembly or data and contributed to final approval of the manuscript. CHT provided financial and administrative support and study material, helped in collection of data and approved final version of the manuscript. MGJ carried out conception and design, financial support, administrative support, provision of study material or patient collection and/or assembly of data, data analysis and interpretation, manuscript writing and final approval of the manuscript. All authors read and approved the final manuscript.

\section{Acknowledgements}

The National Institutes of Health grant RO1 GM087285-01, the Canadian Institutes of Health Research \#123336 funding, the CFI Leader's Opportunity Fund (Project \#25407), the Physician's Services Incorporated Foundation Health Research Grant Program, the Canadian Forces Health Services, and The Healing Foundation/BBA AB Wallace Memorial 2012 Award supported this work.

\section{Author details}

'Plastic Surgery Department and Burn Unit, Vall d'Hebron University Hospital, Universitat Autònoma de Barcelona, Passeig de la Vall d'Hebron 119-129, 08035 Barcelona, Spain. ${ }^{2}$ Ross Tilley Burn Centre and Sunnybrook Research Institute, Sunnybrook Health Sciences Centre, University of Toronto, 2075 Bayview Avenue, Toronto, ON M4N 3M5, Canada. ${ }^{3}$ Gynecology and Obstetrics Department, Sunnybrook Health Sciences Centre, University of Toronto, 2075 Bayview Avenue, Toronto, ON M4N 3M5, Canada. ${ }^{4}$ Canadian Forces Health Services; Trauma, Emergency and Critical Care Program, Sunnybrook Health Sciences Centre, University of Toronto, 2075 Bayview Avenue, Toronto, ON M4N 3M5, Canada.

Received: 31 October 2013 Revised: 12 January 2014

Accepted: 18 February 2014 Published: 24 February 2014

\section{References}

1. Caspersen CJ, Thomas GD, Boseman LA, Beckles GL, Albright AL: Aging, diabetes, and the public health system in the United States. Am J Public Health 2012, 102:1482-1497.

2. Khosrotehrani K: Mesenchymal stem cell therapy in skin: why and what for? Exp Dermatol 2013, 22:307-310
3. Liu S, Yuan M, Hou K, Zhang L, Zheng X, Zhao B, Sui X, Xu W, Lu S, Guo Q: Immune characterization of mesenchymal stem cells in human umbilical cord Wharton's jelly and derived cartilage cells. Cell Immunol 2012, 278:35-44.

4. Hanson SE, Bentz ML, Hematti P: Mesenchymal stem cell therapy for nonhealing cutaneous wounds. Plast Reconstr Surg 2010, 125:510-516.

5. Nekanti U, Rao VB, Bahirvani AG, Jan M, Totey S, Ta M: Long-term expansion and pluripotent marker array analysis of Wharton's jelly-derived mesenchymal stem cells. Stem Cells Dev 2010, 19:117-130

6. Azari O, Babaei H, Drakhsahnfar A, Nematollahi-Mahani SN, Poursahebi R, Moshrefi M: Effects of transplanted mesenchymal stem cells isolated from Wharton's jelly of caprine umbilical cord on cutaneous wound healing: histopathological evaluation. Vet Res Commun 2011, 35:211-222.

7. Moodley Y, Atienza D, Manuelpillai U, Samuel CS, Tchongue J, llancheran S, Boyd R, Trounson A: Human umbilical cord mesenchymal stem cells reduce fibrosis of bleomycin-induced lung injury. Am J Pathol 2009, 175:303-313.

8. Du T, Cheng J, Zhong L, Zhao XF, Zhu J, Zhu YJ, Liu GH: The alleviation of acute and chronic kidney injury by human Wharton's jelly-derived mesenchymal stromal cells triggered by ischemia-reperfusion injury via an endocrine mechanism. Cytotherapy 2012, 14:1215-1227.

9. Tsai PC, Fu TW, Chen YM, Ko TL, Chen TH, Shih YH, Hung SC, Fu YS: The therapeutic potential of human umbilical mesenchymal stem cells from Wharton's jelly in the treatment of rat liver fibrosis. Liver Transp/ 2009, 15:484-495.

10. Xu Y, Huang S, Ma K, Fu X, Han W, Sheng Z: Promising new potential for mesenchymal stem cells derived from human umbilical cord Wharton's jelly: sweat gland cell-like differentiative capacity. J Tissue Eng Regen Med 2012, 6:645-654.

11. Choi M, Lee HS, Naidansaren P, Kim HK, O E, Cha JH, Ahn HY, Yang PI, Shin JC, Joe YA: Proangiogenic features of Wharton's jelly-derived mesenchymal stromal/stem cells and their ability to form functional vessels. In t J Biohem Cell Biol 2013, 45:560-570.

12. Shohara R, Yamamoto A, Takikawa S, Iwase A, Hibi H, Kikkawa F, Ueda M: Mesenchymal stromal cells of human umbilical cord Wharton's jelly accelerate wound healing by paracrine mechanisms. Cytotherapy 2012, 14:1171-1181

13. Kita K, Gauglitz GG, Phan TT, Herndon DN, Jeschke MG: Isolation and characterization of mesenchymal stem cells from the sub-amniotic human umbilical cord lining membrane. Stem Cells Dev 2010, 19:491-501.

14. Arno A, Smith AH, Blit BH, Al-Shehab M, Gauglitz GG, Jeschke MG: Stem cell therapy: a new treatment for burns? Pharmaceuticals 2011, 4:1355-1380.

15. Blit PH, Arno Al, Jeschke MG: Stem cells and tissue engineering in wounds and burns. In Stem Cells Handbook. Edited by Snell S. New York: Springer; 2013:399-410.

16. Amini-Nik S, Glancy D, Boimer C, Whetstone H, Keller C, Alman BA: Pax7 expressing cells contribute to dermal wound repair, regulating scar size through a $\beta$-catenin mediated process. Stem Cells 2011, 29:1371-1379.

17. Falanga V: Wound healing and its impairment in the diabetic foot. Lancet 2005, 366:1736-1743.

18. Bielefeld KA, Amini-Nik S, Alman BA: Cutaneous wound healing: recruiting developmental pathways for regeneration. Cell Mol Life Sci 2013, 70:2059-2081.

19. Jorgensen LN, Kallehave F, Christensen E, Siana JE, Gottrup F: Less collagen production in smokers. Surgeny 1998, 123:450-455.

20. Menke NB, Ward KR, Witten TM, Bonchev DG, Diegelmann RF: Impaired wound healing. Clin Dermatol 2007, 25:19-25.

21. Wasiak J, Cleland H, Campbell F, Spinks A: Dressings for superficial and partial thickness burns. Cochrane Database Syst Rev 2013, 3:CD002106.

22. Li H, Fu X: Mechanisms of action of mesenchymal stem cells in cutaneous wound repair and regeneration. Cell Tissue Res 2012 348:371-377.

23. Hocking AM, Gibran NS: Mesenchymal stem cells: paracrine signaling and differentiation during cutaneous wound repair. Exp Cell Res 2010, 316:2213-2229.

24. Anzalone R, Lo lacono M, Corrao S, Magno F, Loria T, Cappello F, Zummo G, Farina F, La Rocca G: New emerging potentials for human Wharton's jelly mesenchymal stem cells: immunological features and hepatocyte-like differentiative capacity. Stem Cells Dev 2010, 19:423-438. 
25. Bongso A, Fong $C Y$ : The therapeutic potential, challenges and future clinical directions of stem cells from the Wharton's jelly of the human umbilical cord. Stem Cell Rev 2013, 9:226-240.

26. Hass R, Kasper C, Bohm S, Jacobs R: Different populations and sources of human mesenchymal stem cells (MSC): a comparison of adult and neonatal tissue-derived MSC. Cell Commun Signal 2011, 9:12.

27. Kim DW, Staples M, Shinozuka K, Pantcheva P, Kang SD, Borlongan CV: Wharton's jelly-derived mesenchymal stem cells: phenotypic characterization and optimizing their therapeutic potential for clinical applications. Int J Mol Sci 2013, 14:11692-11712.

28. Taghizadeh RR, Cetrulo KJ, Cetrulo CL: Wharton's Jelly stem cells: future clinical applications. Placenta 2011, 32:S311-S315.

29. Rodriguez-Menocal L, Salgado M, Ford D, Van Badiavas E: Stimulation of skin and wound fibroblast migration by mesenchymal stem cells derived from normal donors and chronic wound patients. Stem Cells Transl Med 2012, 1:221-229.

30. Wu Y, Chen L, Scott PG, Tredget EE: Mesenchymal stem cells enhance wound healing through differentiation and angiogenesis. Stem Cells 2007, 25:2648-2659.

31. Luo G, Cheng W, He W, Wang X, Tan J, Fitzgerald M, Li X, Wu J: Promotion of cutaneous wound healing by local application of mesenchymal stem cells derived from human umbilical cord blood. Wound Rep Regen 2010, 18:506-513.

32. Hu JYX, Wang Z, Wang F, Wang L, Gao H, Chen Y, Zhao W, Jia Z, Yan $S$, Wang Y: Long term effects of the implantation of Wharton's jelly-derived mesenchymal stem cells from the umbilical cord for newly-onset type 1 diabetes mellitus. Endocr J 2013, 60:347-357.

33. Rasulov MF, Vasilchenkov AV, Onishchenko NA, Krasheninnikov ME, Kravchenko VI, Gorshenin TL, Pidtsan RE, Potapov IV: First experience of the use bone marrow mesenchymal stem cells for the treatment of a patient with deep skin burns. Bull Exp Biol Med 2005, 139:141-144.

34. Balasubramanian S, Venugopal P, Sundarrai J, Zakaria Z, Majumdar AS, Ta M: Comparison of chemokine and receptor gene expression between Wharton's jelly and bone marrow-derived mesenchymal stromal cells. Cythotherapy 2012, 14:26-33.

35. Christodoulou I, Kolisis FN, Papaevangeliou D, Zoumpourlis V: Comparative evaluation of human mesenchymal stem cells of fetal (Wharton's jelly) and adult (adipose tissue) during prolonged in vitro expansion: considerations for cytotherapy. Stem Cells Int 2013, 2013:246134.

doi:10.1186/scrt417

Cite this article as: Arno et al:: Human Wharton's jelly mesenchymal stem cells promote skin wound healing through paracrine signaling. Stem Cell Research \& Therapy 2014 5:28.

\section{Submit your next manuscript to BioMed Central and take full advantage of:}

- Convenient online submission

- Thorough peer review

- No space constraints or color figure charges

- Immediate publication on acceptance

- Inclusion in PubMed, CAS, Scopus and Google Scholar

- Research which is freely available for redistribution 